\title{
Reconstrucción ósea de defectos craneales secundarios a traumatismo con implantes personalizados
}

\section{Cranial bone reconstruction with customized implants after trauma}

\author{
Ricardo Cienfuegos ${ }^{1,2 *}$, Gerardo Fernández ${ }^{2,3}$, Aída Cruz y Eduardo Sierra ${ }^{4}$ \\ 'División de Educación, Unidad Médica de Alta Especialidad (UMAE), Traumatología y Ortopedia Lomas Verdes, Instituto Mexicano del Seguro \\ Social (IMSS); ${ }^{2}$ Centro Médico ABC Observatorio; ${ }^{3}$ Servicio de Cirugía Plástica, Instituto Nacional de Pediatría; ${ }^{4}$ UMAE Traumatología y Ortopedia \\ Lomas Verdes, IMSS. Ciudad de México, México
}

\begin{abstract}
Resumen
Introducción: Los defectos craneales secundarios a traumatismos son frecuentes. Por lo común se reparan de forma secundaria por sintomatología como el síndrome del paciente trepanado, por protección cerebral y por el aspecto cosmético. Históricamente se han utilizado diversos materiales para la reconstrucción. Casos clínicos: Se presentan cinco casos de pacientes reconstruidos con implantes personalizados de polieteretercetona (PEEK) o polimetilmetacrilato poroso (PMMA). Las localizaciones afectaron el frontal, el borde orbitario superior y el techo orbitario en cuatro casos, y la porción lateral del frontal, la zona temporoparietal y el borde del occipital en un caso. La reconstrucción en cuatro pacientes fue entre 6 y 12 meses después de la lesión, y en un caso después de 25 años. En dos casos se requirió expansión tisular antes de colocar el implante. Resultados: Cuatro pacientes evolucionaron favorablemente, con mejoría de los síntomas neurológicos, forma y contorno adecuados, así como un proceso de cicatrización adecuada de los colgajos de piel cabelluda. Un paciente presentó infección por Staphylococcus aureus, atribuida a la presencia de un mucocele y una fístula de la vía aérea a la cavidad craneal, lo que hizo necesario retirar el implante. Conclusiones: Los implantes personalizados son un recurso útil para defectos óseos craneales. Brindan resultados satisfactorios desde el punto de vista funcional y cosmético. Deben tomarse precauciones respecto al tratamiento de las lesiones que afecten el seno frontal, para evitar la comunicación entre la vía aérea y la cavidad craneal.
\end{abstract}

PALABRAS CLAVE: PEEK. PMMA. Polieteretercetona. Polimetilmetacrilato. Procedimientos quirúrgicos reconstructivos. Prótesis e implantes.

\begin{abstract}
Introduction: Cranial defects due to trauma are frequent. They are usually repaired in a secondary fashion due to features such as syndrome of the trephined, for brain protection and for cosmetic purposes. Historically, various materials have been used for reconstruction. Case reports: Five cases of patients reconstructed with customized polyetheretherketone (PEEK) 0 polymethyl methacrylate (PMMA) implants are presented. Defects involved the frontal bone, superior orbital rim and orbital roof in four cases, and the lateral area of the frontal bone, the temporoparietal area and the border of the occipital bone in one. In four patients, reconstruction took place between 6 and 12 months after the injury; in one patient, after 25 years. Two cases required tissue expansion before placing the implant. Results: Four patients evolved favorably, with improvement in neurologic symptoms and adequate shape and contour, plus adequate healing of the scalp flaps. One patient had an infection due to Staphylococcus aureus, attributed to a mucocele and fistula between the airway and the cranial cavity, leading to removal of
\end{abstract}

\author{
Correspondencia: \\ *Ricardo Cienfuegos \\ Sur 136, 116-111 \\ Col. Las Américas, Del. Álvaro Obregón \\ C.P. 01120, Ciudad de México, México \\ E-mail: rcienfuegos@usa.net
}

Fecha de recepción: 22-03-2018

Fecha de aceptación: 26-04-2018

DOI: 10.24875/CIRU.M18000042
Cir Cir. 2018;86:289-295

Contents available at PubMed www.cirugiaycirujanos.com 
the implant. Conclusions: Customized implants are a useful resource for cranial defects. They offer satisfactory results, both functional and cosmetic. Precautions should be taken in treating injuries that involve the frontal sinus, to ensure there is no communication between the airway and the cranial cavity.

KEY WORDS: PEEK. PMMA. Polyetheretherketone. Polymethyl methacrylate. Reconstructive surgical procedures. Prostheses and implants.

\section{Introducción}

Los defectos esqueléticos craneales secundarios a traumatismos son comunes, debido a la gran frecuencia de accidentes viales, violencia urbana e incluso por deportes de contacto. Algunos defectos óseos también pueden deberse a infecciones, procedimientos quirúrgicos 0 tumores. El tratamiento neuroquirúrgico de los traumatismos craneales incluye craneotomías y retiro de segmentos óseos, por fracturas expuestas y lesiones intracraneales como hematomas. Los defectos óseos se reparan de forma secundaria, ya que en muchos casos no está indicada la reparación primaria por la posibilidad de infección. Ante este panorama, la reconstrucción craneal tiene como finalidad tanto el aspecto funcional como el cosmético. El aspecto funcional considera el alivio de la sintomatología neurológica debida al llamado «síndrome del colgajo hundido» 0 «síndrome del paciente trepanado", así como la protección del contenido intracraneal'. También debe considerarse la restitución del aspecto cosmético, primordialmente si el defecto óseo se encuentra cerca de la cara, como sucede con los defectos frontales (Fig. 1). Para la reparación de las pérdidas óseas del cráneo pueden utilizarse materiales autólogos, heterólogos y aloplásticos; estos últimos incluyen metales y plásticos.

El objetivo de este trabajo es presentar el resultado de cinco casos tratados mediante reconstrucción de la bóveda craneal con implantes aloplásticos personalizados, cuatro de polieteretercetona (PEEK) y uno de polimetilmetacrilato poroso (PMMA). Estos tipos de materiales aloplásticos ofrecen como ventaja su permanencia por tiempo indefinido; brindan estabilidad mecánica, adaptabilidad a la zona por reconstruir, biocompatibilidad y protección al contenido intracraneal, además de un buen resultado cosmético que incluye restaurar la simetría. Pueden esterilizarse en repetidas ocasiones sin que se alteren sus propiedades, y son compatibles con la resonancia magnética y la tomografía computarizada. Otro beneficio por considerar es la mejora en la sintomatología asociada al síndrome del colgajo hundido ${ }^{2,3}$.

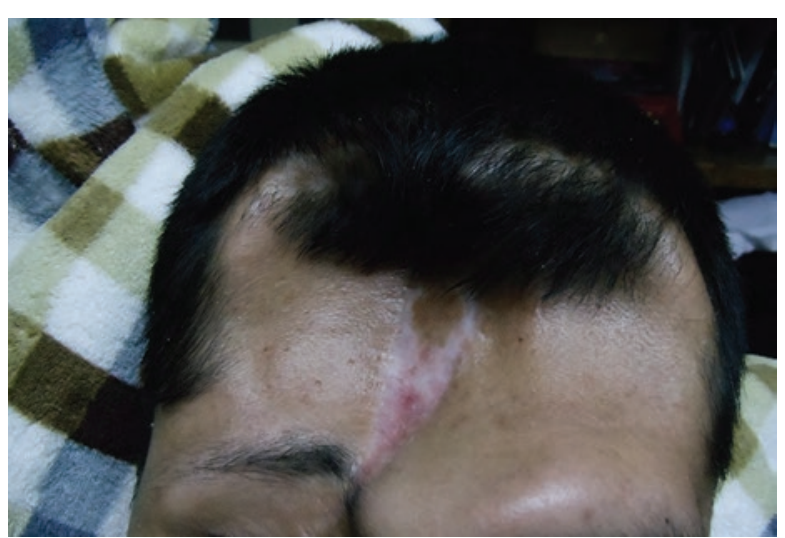

Figura 1. Defecto frontal y del borde orbitario superior, y hundimiento del colgajo frontal (caso 4, Tabla 1).

\section{Casos clínicos}

Se hizo una revisión de pacientes sometidos a tratamiento quirúrgico por los autores para reconstrucción de defectos óseos craneales que fueron atendidos entre 2008 y 2016, tanto en un hospital público (Unidad Médica de Alta Especialidad Hospital de Traumatología y Ortopedia Lomas Verdes) como en uno privado (Centro Médico $A B C$ ) de la Ciudad de México.

Los defectos óseos eran secundarios a traumatismos craneoencefálicos o craneofaciales, así como a craneotomías posteriores al traumatismo. Los pacientes se evaluaron tanto en forma clínica como por imagenología, mediante tomografía computarizada de cortes finos $(1 \mathrm{~mm})$.

Se trató con implantes craneales fabricados en forma personalizada a cinco pacientes varones. Las edades iban de los 15 a los 50 años, con una media de 29.2 años. Las zonas por reconstruir fueron la región frontal asociada con el borde supraorbitario y parcialmente el techo orbitario en cuatro casos, y la porción lateral del frontal, la zona temporoparietal y el borde del occipital en un caso. Cuatro de los cinco pacientes habían sufrido también fracturas faciales. Para la fabricación del implante personalizado, en todos los casos se realizó una tomografía computarizada con cortes a $1 \mathrm{~mm}$, que se envió al fabricante. Se colocaron cuatro implantes de PEEK (DePuy Synthes, West Chester, PA) y uno de PMMA (Zimme Biomet, Warsaw, IN) (Tabla 1). 
Para efectuar la reconstrucción, se consideró conveniente que los pacientes tuvieran entre 6 y 12 meses de evolución postoperatoria con el defecto óseo craneal. Se analizó clínicamente la cicatrización de las heridas quirúrgicas y que no hubiera presencia de fístulas, áreas cruentas ni infección. Se incluyó un

Tabla 1. Características de los cinco casos e imagen de los implantes personalizados

\begin{tabular}{|c|c|c|c|c|c|c|}
\hline Caso & $\begin{array}{l}\text { Sexo y } \\
\text { edad }\end{array}$ & $\begin{array}{l}\text { Localización y } \\
\text { dimensiones del defecto }\end{array}$ & $\begin{array}{l}\text { Material } \\
\text { del } \\
\text { implante }\end{array}$ & Tiempos quirúrgicos & Complicaciones & Imagen del implante \\
\hline 1 & $\begin{array}{l}\text { Masculino } \\
24 \text { años }\end{array}$ & $\begin{array}{l}\text { Frontoorbitario izquierdo } \\
(13 \times 10 \mathrm{~cm})\end{array}$ & PEEK & $\begin{array}{l}\text { Dos: } \\
\text { - Expansor tisular } \\
\text { parieto- occipital } \\
\text { - Retiro de expansor y } \\
\text { colocación de implante }\end{array}$ & No & \\
\hline 2 & $\begin{array}{l}\text { Masculino } \\
15 \text { años }\end{array}$ & $\begin{array}{l}\text { Frontal, parietal, temporal y } \\
\text { occipital izquierdos } \\
(20 \times 16 \mathrm{~cm})\end{array}$ & PEEK & Uno & No & \\
\hline 3 & $\begin{array}{l}\text { Masculino } \\
27 \text { años }\end{array}$ & $\begin{array}{l}\text { Techo orbitario derecho } \\
\text { Frontal, parietal y temporal } \\
\text { derechos } \\
(12.4 \times 12 \mathrm{~cm})\end{array}$ & PMMA & Uno & $\begin{array}{l}\text { Sí: colección } \\
\text { frontal por } \\
\text { mucocele y fístula } \\
\text { a los } 11 \text { meses. } \\
\text { Retiro de implante; } \\
\text { cultivo positivo } \\
\text { para } S \text {. aureus }\end{array}$ & \\
\hline 4 & $\begin{array}{l}\text { Masculino } \\
30 \text { años }\end{array}$ & $\begin{array}{l}\text { Frontal, parietal y temporal } \\
\text { bilateral } \\
(18.6 \times 12 \mathrm{~cm})\end{array}$ & PEEK & $\begin{array}{l}\text { Tres: } \\
\text { Osteotomía y reconstrucción } \\
\text { de cigoma derecho; } \\
\text { colocación de expansor } \\
\text { tisular parieto- occipital } \\
\text { Retiro de expansor } \\
\text { Colocación de implante }\end{array}$ & No & \\
\hline 5 & $\begin{array}{l}\text { Masculino } \\
50 \text { años }\end{array}$ & $\begin{array}{l}\text { Frontal y supraorbitario } \\
\text { izquierdo } \\
(7.7 \times 2.8 \mathrm{~cm})\end{array}$ & PEEK & $\begin{array}{l}\text { Uno: } \\
\text { Osteotomías y fijación de } \\
\text { ambos cigomas; malla en } \\
\text { ambos pisos orbitarios; } \\
\text { suspensión de tejidos } \\
\text { blandos de tercio medio; } \\
\text { colocación de implante }\end{array}$ & No & \\
\hline
\end{tabular}


caso de secuelas de politraumatismo, con fracturas faciales y pérdida ósea de la pared anterior del seno frontal y parcialmente del techo orbitario del lado izquierdo, que tenía más de 25 años de evolución.

Los procedimientos quirúrgicos se llevaron a cabo bajo anestesia general y los implantes se colocaron a través de la cicatriz del acceso coronal previo (Fig. 2), con excepción del paciente más joven (de 15 años), a quien se le colocó el implante a través del acceso quirúrgico lateral de cráneo del tratamiento inicial. La reconstrucción de la pérdida craneal con el implante personalizado se hizo en un solo tiempo en tres de los cinco; en el cuarto caso, en dos tiempos, y en el quinto caso, en tres tiempos quirúrgicos. En estos dos últimos pacientes se ejecutaron procedimientos secuenciales: inicialmente se colocó en ambos un expansor tisular a fin de mejorar la disponibilidad de piel para la reconstrucción, ya que el colgajo de piel cabelluda estaba retraído y se consideró insuficiente para cubrir el área reconstruida con el implante (Fig. 3). Ambos expansores (de válvula remota) se ubicaron en la región parietooccipital. Uno de estos casos presentó celulitis en el área de expansión, por lo que fue necesario retirar el expansor, además de dar tratamiento antimicrobiano. El implante personalizado se colocó 3 meses después, para evitar la retracción de la piel cabelluda, tras comprobar la ausencia de infección.

Los implantes se fijaron al área receptora con placas y tornillos de titanio de $1.5 \mathrm{~mm}$ de diámetro. Se colocaron puntos de sutura en la duramadre, que se extrajeron por los orificios del implante y se anudaron sobre este, para adosar la meninge a la cara interna del implante (Fig. 4). El armado del acceso coronal se hizo en dos planos, con nailon de calibre 3-0 y 4-0, respectivamente. Se utilizó en todos los pacientes un drenaje cerrado con sistema de vacío durante 3 días, para evitar una colección serohemática subcutánea, además de favorecer la adherencia del colgajo al lecho y también al implante. Las suturas de la piel cabelluda se retiraron 8 días después de la intervención. En todos los casos se administró antimicrobiano, el cual se inició antes de la intervención y se continuó durante 7 días.

Como control postoperatorio se practicó tomografía computarizada en todos los casos, además de vigilar la evolución clínica.

\section{Resultados}

Se reconstruyeron las pérdidas óseas en el cráneo de cinco pacientes, todas de origen traumático,

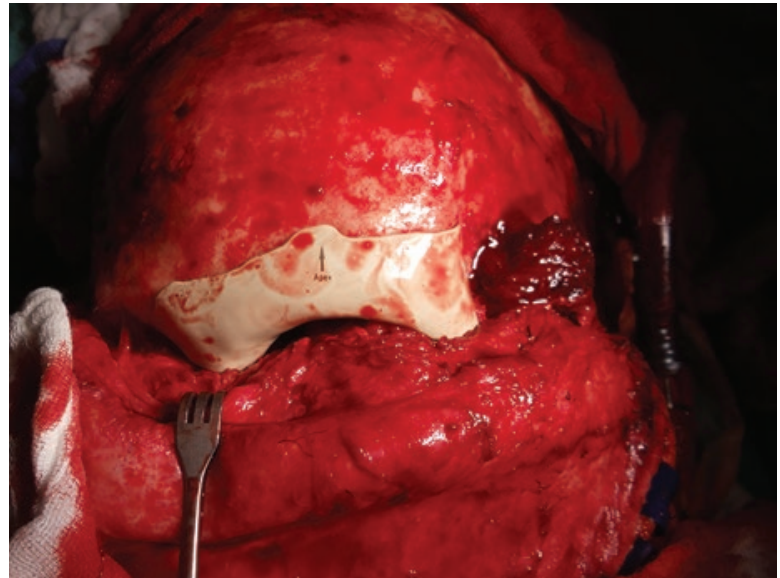

Figura 2. Acceso coronal para colocar el implante por pérdida frontoorbitaria (caso 5, véase la tabla 1).

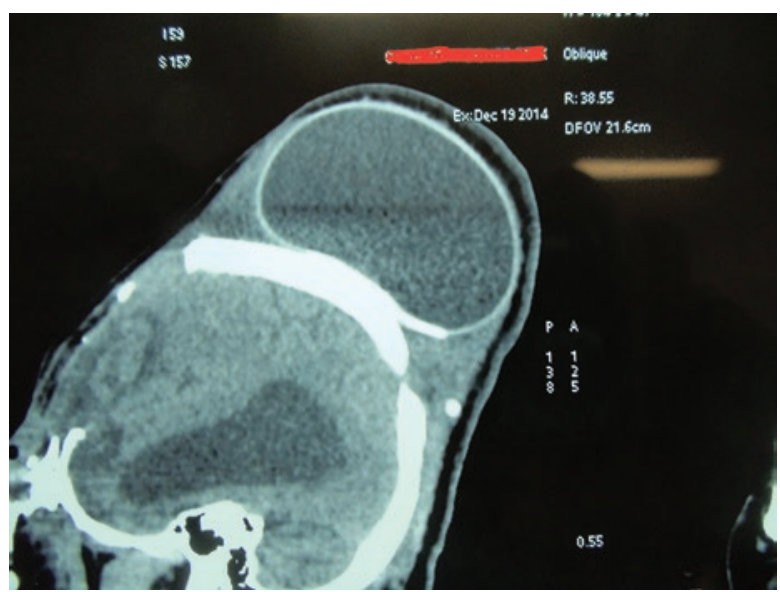

Figura 3. Imagen tomográfica del expansor tisular sobre la porción posterior del cráneo.

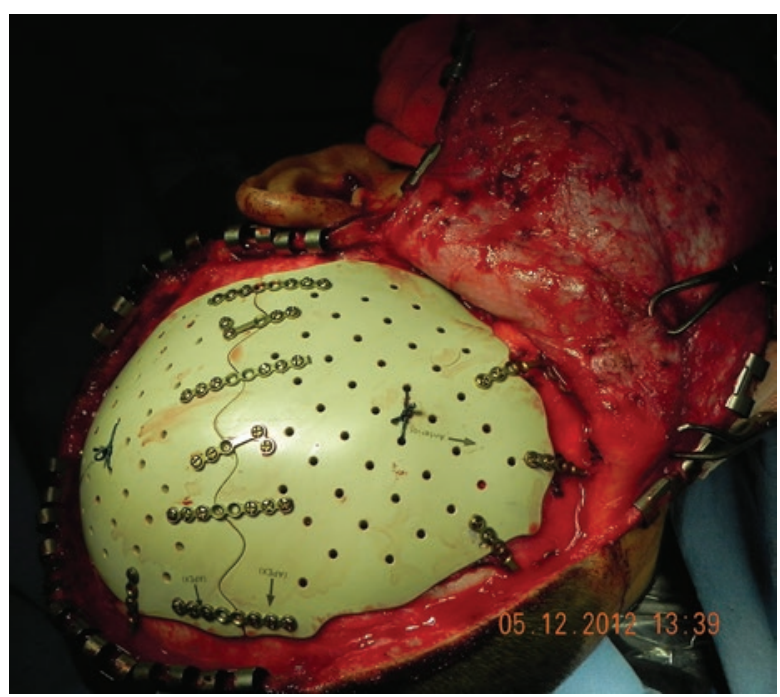

Figura 4. Implante personalizado en dos piezas, colocado en un defecto lateral del cráneo, de frontal a occipital. Nótese la colocación de suturas para aproximar la duramadre al implante (caso 2, Tabla 1). 
mediante el uso de implantes personalizados, fabricados a la medida a partir de una tomografía computarizada. Se utilizaron cuatro implantes de PEEK y uno de PMMA. Todos los defectos se reconstruyeron en fase de secuela, no menos de 6 a 12 meses después del tratamiento inicial. Uno de los casos (el paciente de mayor edad) se sometió a reconstrucción 25 años después del accidente causal.

Como ya se mencionó, en tres de los cinco casos la reconstrucción se efectuó en un solo tiempo quirúrgico. En el paciente tratado en dos tiempos quirúrgicos, en la primera intervención se colocó el expansor tisular. Este se fue llenando gradualmente hasta obtener un volumen suficiente, y entonces se planeó el retiro del expansor y la colocación del implante en el segundo tiempo quirúrgico. Ambos procedimientos se llevaron a cabo sin complicaciones (Fig. 5).

En el paciente tratado en tres tiempos quirúrgicos, en la primera intervención se realizó osteotomía con reducción y fijación del cigoma, y reconstrucción del piso y la pared medial de la órbita (por secuelas de fracturas del cigoma no tratadas en la atención inicial), además de la colocación del expansor tisular. Cuando faltaban pocos días para el retiro del expansor y la colocación del implante en el segundo tiempo quirúrgico, se presentó un cuadro de hiperemia cutánea, la cual se catalogó como celulitis, que hizo necesario retirar el expansor. El estudio tomográfico no reveló colecciones subcutáneas ni en la cavidad intracraneal. Al momento de retirar el expansor no se encontró material purulento, y se obtuvo una buena respuesta clínica con la utilización de clindamicina por 10 días. Se solicitaron cultivos del expansor y los tejidos adyacentes, que fueron negativos para desarrollo bacteriano. Para evitar retracción del colgajo expandido, apoyados en el resultado de los cultivos transoperatorios y la evolución del paciente, se decidió efectuar la reconstrucción craneal con el implante 3 meses después de retirar el expansor tisular, en un tercer tiempo quirúrgico. El paciente evolucionó sin complicaciones ulteriores (Fig. 6).

Los accesos quirúrgicos cicatrizaron sin complicaciones en todos los casos.

En los cinco casos las dimensiones del implante fueron idóneas, ya que no hubo necesidad de modificar el área receptora ni el implante al momento de la colocación. La fijación de todos los implantes en el área receptora con placas y tornillos de titanio de $1.5 \mathrm{~mm}$ de diámetro se llevó a cabo sin mayor dificultad y evolucionó sin complicaciones.

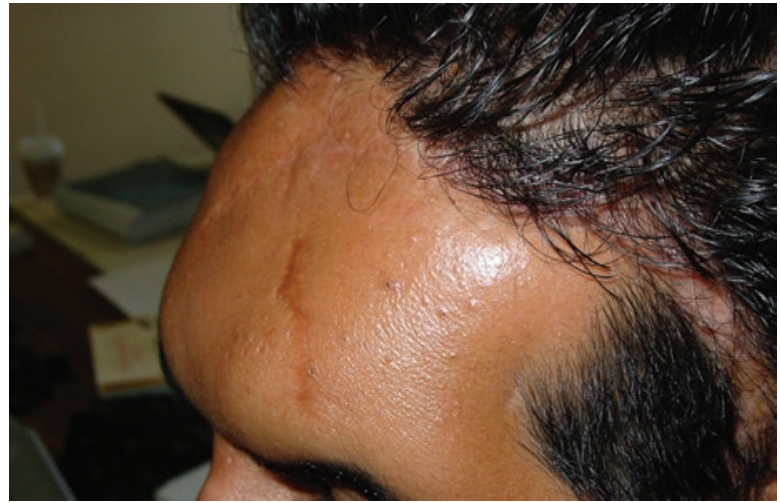

Figura 5. Evolución a los 18 meses de la reconstrucción con implante frontoorbitario izquierdo (caso 1, Tabla 1).

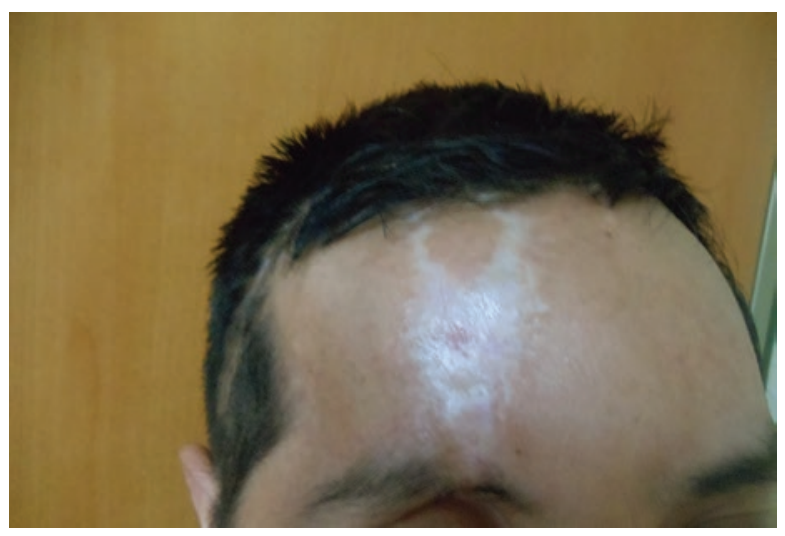

Figura 6. Imagen postoperatoria del caso 4 a los 24 meses de evolución postoperatoria. Obsérvese el contorno de la zona frontoorbitaria (Fig. 1 y Tabla 1).

A los cuatro meses de la colocación del implante personalizado de PMMA, el paciente presentó un cuadro clínico caracterizado por lesiones cutáneas frontales (pápulas) y fluctuación subcutánea de la región reconstruida. La tomografía computarizada reveló la presencia de una colección en la región nasofrontal compatible con un mucocele. Se decidió retirar el implante. Durante la operación para el retiro se encontró material purulento (4 ml), que se envió para cultivo. Se descubrió un defecto óseo en la porción baja del frontal que comunicaba con la nariz. El defecto óseo se trató mediante abrasión de la mucosa, escarificación ósea y relleno con músculo y esponja de gelatina estéril (Gelfoam ${ }^{\circledR}$ ), y se cubrió con un pequeño colgajo de periostio local. El cultivo reportó la presencia de Staphylococcus aureus, por lo que se administró vancomicina con imipenem durante 10 días. El estado postoperatorio al cabo de 10 meses es satisfactorio. Además de una evolución clínica favorable, los estudios tomográficos muestran obliteración de la fístula; no se observa persistencia del defecto óseo ni 
formación de mucocele. El paciente está en espera de una nueva reconstrucción craneal.

Nuestros pacientes se han sometido a seguimientos largos, de hasta 24 meses, tiempo durante el cual se ha podido constatar una cicatrización adecuada, estabilidad del implante y que la forma del área reconstruida recupera la simetría y se mantiene sin alteraciones (salvo en el caso del implante retirado).

En los pacientes sometidos a craniectomía se ha descrito el llamado «síndrome del paciente trepanado" o "síndrome del colgajo hundido" (syndrome of the trephined o sinking skin flap syndrome), caracterizado por déficits motores, cognitivos y de lenguaje, cefalea y otros síntomas ${ }^{4}$. La reconstrucción de los defectos de espesor total del cráneo mejora estas manifestaciones ${ }^{5}$. Cuatro de nuestros pacientes habían sufrido pérdida ósea craneal de espesor total y presentaban sintomatología compatible con este síndrome (cefaleas, mareos, trastornos de la marcha). No se realizó una medición cuantitativa de dichas alteraciones neurológicas en el preoperatorio. Sin embargo, en el postoperatorio los pacientes refirieron cualitativamente una disminución notoria de la intensidad de la cefalea y de la presencia de mareo, así como mejoría en la marcha. Estos cambios fueron evidentes a partir de 3 meses de postoperatorio.

\section{Discusión}

La reconstrucción de defectos craneales es un procedimiento que se ha practicado a lo largo de los siglos. Se han usado muy diversos materiales, que van desde el hueso de perro o el hueso autólogo hasta el oro $^{2}$. La reconstrucción tiene como objetivo fundamental la protección del cerebro, el aspecto estético y también, dentro del ámbito funcional, disminuir las manifestaciones del síndrome del colgajo hundido ${ }^{2,4-6}$.

Actualmente se cuenta con una gran variedad de materiales biológicos y aloplásticos para la reconstrucción de las pérdidas óseas craneales. Puede utilizarse hueso autólogo, aunque tiene como desventajas la posibilidad de reabsorción, un tiempo quirúrgico más prolongado, sangrado y morbilidad para el área donadora ${ }^{2,3}$.

Entre los materiales aloplásticos está la malla de titanio, con las ventajas de ser biocompatible y maleable, pero con la desventaja de no brindar un buen contorno en áreas de dimensiones considerables o bien en zonas como la región frontal, además de que puede ocasionar artefactos en los estudios de imagenología ${ }^{3}$. El metilmetacrilato se ha utilizado ampliamente, pero es difícil amoldarlo y puede causar lesiones en los tejidos adyacentes debido a la reacción exotérmica producida durante su polimerización ${ }^{3}$.

El uso de los implantes diseñados por computadora no es nuevo, ya que se inició en 19957. Este tipo de implantes, que pueden ser tanto de PEEK como de PMMA, ofrecen múltiples ventajas. Los implantes de PEEK y PMMA pueden perforarse con una broca e insertárseles tornillos para fijarlos; de igual manera, en caso de utilizar tornillos autoperforantes, pueden insertarse estos sin problemas. Cabe destacar también que se acorta el tiempo quirúrgico y se reduce la morbilidad del área donadora. A diferencia del hueso autólogo, no es necesario esperar la consolidación de un injerto ni hay el riesgo de que se reabsorba. Otro beneficio es la resistencia, la restitución del contorno del cráneo reconstruido y la restauración de la simetría, situación muy notoria cuando el defecto se localiza en la región frontal.

Tres requisitos de importancia capital son un lecho con vascularidad adecuada, una cubierta cutánea estable y suficiente, y que no haya infección local. Sea cual fuere la gravedad de la lesión, el tratamiento inicial debe contribuir a estas condiciones con miras a la reconstrucción futura. Si hubiera retracción del colgajo que dificulte cubrir el implante o entrañe el riesgo de exposición, puede recurrirse a la expansión tisular previa. En caso de que el defecto se comunique con el seno frontal, es fundamental aislar este último para reducir el riesgo de infección por continuidad con las vías aéreas.

En nuestra serie encontramos que no se requirió ningún ajuste de los implantes al colocarlos en el área receptora. En la experiencia que hemos tenido, a fin de disminuir el espacio muerto entre el implante y la superficie dural colocamos algunas suturas (riendas) para adosar la duramadre a la cara interna del implante. El uso de drenajes con succión ayuda a evitar la formación de hematomas y seromas, y además favorece la adherencia del colgajo de piel cabelluda al lecho constituido por el implante.

Consideramos que la infección que presentó el paciente reconstruido con el implante de PMMA guardó relación directa con el mucocele y la fístula existente, debido a la comunicación entre la parte alta de la vía aérea superior y el seno frontal. Este punto debe tenerse muy presente al tratar las fracturas que afectan al seno fronta|8,9. Diez meses después del retiro del implante, el paciente se encuentra asintomático, con evolución favorable y sin datos imagenológicos de recidiva del trayecto fistuloso. A pesar de ello, se considera prudente esperar algunos meses más para 
intentar una nueva reconstrucción de la región frontal.

Sería importante crear conciencia sobre el cuadro clínico del «síndrome del colgajo hundido» o «síndrome del paciente trepanado" entre los especialistas que atienden pacientes con defectos de la bóveda craneana. Una posible línea de investigación consistiría en documentar detalladamente las manifestaciones del síndrome, así como su mitigación o resolución gracias a los procedimientos reconstructivos. Otra línea pertinente buscaría establecer los criterios específicos sobre qué tipo de defectos son susceptibles de reconstrucción con mallas de titanio, implantes prefabricados o injertos óseos, tanto desde el punto de vista de dimensiones como, posiblemente, de su localización anatómica.

\section{Conclusiones}

La reconstrucción craneal constituye una necesidad para los pacientes sometidos a craniectomía con pérdida ósea, no solo para fines cosméticos, sino también para mejorar la sintomatología neurológica secundaria a la pérdida ósea, denominada "síndrome del paciente trepanado» 0 "síndrome del colgajo hundido», además de brindar protección al cerebro.

Según algunos autores, la reconstrucción de defectos craneales con implantes personalizados fabricados de PEEK o de PMMA es una buena solución; no hay evidencia suficiente que demuestre la superioridad de uno de estos materiales sobre el otro ${ }^{10}$. Nuestra serie es pequeña, de cinco casos, de los cuales solo uno presentó complicaciones por infección, atribuible a una fístula que conectaba la parte alta de la cavidad nasal con el seno frontal y a la presencia de un mucocele; ese caso fue el único en el que se utilizó un implante de PMMA. Otro punto por considerar es el espacio muerto entre la superficie del cerebro y la cara interna del implante; se recomienda que este espacio no sea mayor de $2 \mathrm{~cm}$, para evitar la formación de hematomas y seromas susceptibles de infectarse ${ }^{11}$. Hemos logrado resolver esta situación mediante la colocación de suturas para adosar la duramadre al implante.
La tecnología de impresión tridimensional aplicada a la cirugía del esqueleto craneofacial ha mejorado continuamente desde su invención en la década de $1980^{12}$. Hoy existe la opción de utilizar prótesis fabricadas a la medida, a partir de una tomografía computarizada, para reparar las pérdidas óseas. Los materiales tienen mayor biocompatibilidad, son ligeros y resistentes, y pueden fijarse de manera estable con placas y tornillos en el área receptora. En el caso específico de los implantes personalizados, constituyen una solución integral y práctica, que brinda excelentes resultados cosméticos y funcionales para este tipo de reconstrucciones. Aún falta definir con mayor precisión las indicaciones específicas para la reconstrucción mediante implantes personalizados.

\section{Bibliografía}

1. Manson PN, Crawley WA, Hoopes JE. Frontal cranioplasty: risk factors and choice of cranial vault reconstructive material. Plast Reconstr Surg. 1986;77:888-900.

2. Jonkergouw J, van de Vijfeijken SECM, Nout E, Theys T, van de Casteele $\mathrm{E}$, Folkersma $\mathrm{H}$, et al. Outcome in patient-specific PEEK cranioplasty: a two-center cohort study of 40 implants. J Craniomaxillofac Surg. 2016;44:1266-72.

3. Alonso-Rodríguez E, Cebrián JL, Nieto MJ, del Castillo JL, Hernández-Godoy J, Burgueño M. Polyetheretherketone custom-made implants for craniofacial defects: report of 14 cases and review of the literature. J Craniomaxillofac Surg. 2015;43:1232-8.

4. Ashayeri K, Jackson EM, Huang J, Brem H, Gordon CR. Syndrome of the trephined: a systematic review. Neurosurgery. 2016;79:525-34.

5. Isago T, Nozaki M, Kikuchi Y, Honda T, Nakazawa H. Sinking skin flap syndrome: a case of improved cerebral blood flow after cranioplasty. Ann Plast Surg. 2004;53:288-92.

6. Zegers T, ter Laak-Poort M, Koper D, Lethaus B, Kessler P. The therapeutic effect of patient-specific implants in cranioplasty. J Craniomaxillofac Surg. 2017;45:82-6.

7. Gerbino G, Zavattero E, Zenga F, Bianchi FA, Garzino-Demo P, Berrone S. Primary and secondary reconstruction of complex craniofacial defects using polietheretherketone custom-made implants. J CraniomaxiIlofac Surg. 2015;43:1356-63.

8. Rodríguez ED, Stanwix MG, Nam AJ, St Hilaire H, Simmons OP, Christy MR, et al. Twenty-six year experience treating frontal sinus fractures: a novel algorithm based on anatomical fracture pattern and failure of conventional techniques. Plast Reconstr Surg. 2008;122:1850-66.

9. Zubillaga Rodríguez D, Lora Pablos MI, Falguera Uceda R, Díez Lobato G, Sánchez Aniceto G. Frontal sinus obliteration after trauma: analysis of bone regeneration for two selected methods. Int $\mathrm{J}$ Oral Maxillofac Surg. 2014;43:827-33.

10. Iaccarino C, Viaroli E, Fricia M, Serchi E, Poli T, Servadei F. Preliminary results of a prospective study on methods of cranial reconstruction. J Oral Maxillofac Surg. 2015;73:2375-8.

11. Kumar AR, Tantawi D, Armonda R, Valerio I. Advanced cranial reconstruction using intracranial free flaps and cranial bone grafts: an algorithmic approach developed from the modern battlefield. Plast Reconstr Surg. 2012;130:1101-8.

12. Jacobs CA, Lin AY. A new classification of three-dimensional printing technologies: systematic review of three-dimensional printing for patient-specific craniomaxillofacial surgery. Plast Reconstr Surg. 2017; 139:1211-20. 